\title{
The Reflection Principle in Complex Space
}

\author{
FRANCINE MEYLAN
}

1. Introduction. Let $M$ and $M^{\prime}$ be two germs of real analytic generic submanifolds at 0 of codimension $d \geq 1$ in $\mathbb{C}^{n}, n \geq 1$, and $\mathcal{H}$ (a germ at 0 of) a holomorphic map defined in a (germ of) wedge $\mathcal{W}$ with edge $M$, smooth in (the germ of) $\mathcal{W} \cup M$, and mapping $M$ into $M^{\prime}$, with $\mathcal{H}(0)=0$. We say that the reflection principle holds if $\mathcal{H}$ is the restriction of a holomorphic mapping in a neighborhood of 0 from $\mathbb{C}^{n}$ into $\mathbb{C}^{n}$. In the complex plane, by the classical Schwarz reflection principle, the reflection principle holds. The first results in higher dimension were due to H. Lewy [14 ] and S. Pinčuk [18] , who proved independently that the reflection principle holds if $M$ and $M^{\prime}$ are strictly pseudoconvex, and $\mathcal{H}$ is a diffeomorphism from $M$ to $M^{\prime}$. These results have been extended by Baouendi, Jacobowitz and Treves [2] for $M$ and $M^{\prime}$, germs of real analytic generic submanifolds at 0 of codimension $d \geq 1$, satisfying an algebraic condition, and $\mathcal{H}$ a diffeomorphism from $M$ to $M^{\prime}$. Other results on the reflection principle for $d=1$ have been obtained by Baouendi and Rothschild [3], [4], [5], Bell [6], Diederich and Fornaess [9], Diederich and Webster [10], the author [16], [17], as well as by other mathematicians. In [3] and [4] a reflection principle is obtained in $\mathbb{C}^{n}$ for $M$ and $M^{\prime}$ germs of real analytic hypersurfaces at 0 of finite type. The mapping they consider is no longer a diffeomorphism.

In this paper, we address the question of extending the reflection principle in $\mathbb{C}^{n}$ for $M$ and $M^{\prime}$ germs of real analytic generic submanifolds of codimension $d \geq 1$ to a new class of mappings, generalizing the results obtained in [2], [3] and [4].

Let $M$ be a germ of a real analytic generic submanifold of codimension $d \geq 1$ at 0 . After a local holomorphic change of coordinates, we can assume that there exists $\Omega$, a sufficiently small open neighborhood of 0 in $\mathbb{C}^{n}$, such that $M$ is given by

$$
\operatorname{Im} w=\varphi(z, \bar{z}, \operatorname{Re} w),
$$

with $z \in \mathbb{C}^{n-d}, w \in \mathbb{C}^{d}, \varphi=\left(\varphi_{1}, \ldots, \varphi_{d}\right)$, where $\varphi_{j}, 1 \leq j \leq d$, are real valued convergent power series and $\varphi(z, 0, w) \equiv 0$ (cf. [2]). Such a choice of coordinates is 
called normal coordinates. Recall that $M$ is essentially finite at 0 if the following is true

$$
\varphi(z, \zeta, 0)=0 \quad \text { for all } \zeta \in \mathbb{C}^{n-d} \Longrightarrow z=0 .
$$

This definition is independent of the choice of normal coordinates (cf. [2] ). Write $\varphi_{j}(z, \zeta, 0)=\sum a_{\alpha}^{j}(z) \zeta^{\alpha}$. By the Nullstellensatz, the definition above is equivalent to say that

$$
\text { ess. type } M=\operatorname{dim}_{\mathbb{C}} \frac{\mathbb{C}[[z]]}{\left(a_{\alpha}^{j}(z)\right)}<\infty,
$$

where $\left(a_{\alpha}^{j}(z)\right)$ is the ideal in the ring of formal power series $\mathbb{C}[[z]]$ generated by all the $a_{\alpha}^{j}(z)$. We shall see that the number ess. type $M$ is independent of the choice of normal coordinates. Note that if $d=1, M$ essentially finite implies $M$ of finite type. This is no longer true in higher codimension (cf. [2]).

Let $\Gamma$ be a non-empty open convex cone with vertex at the origin in $\mathbb{R}^{d}$, and let $\mathcal{W}_{\Gamma}$ be the wedge with edge $M$ in the direction $\Gamma$ such that $\mathcal{H}$ is (a germ at 0 of) a holomorphic map in (the germ of) $\mathcal{W}_{\Gamma}$, smooth in (the germ of) $\mathcal{W}_{\Gamma} \cup M$, valued in $\mathbb{C}^{n}$, and satisfying $\mathcal{H}(M) \subset M^{\prime}$, where $M^{\prime}$ is another germ of real analytic generic submanifold of codimension $d$ at 0 in $\mathbb{C}^{n}$, also given in normal coordinates. We shall always assume $\mathcal{H}(0)=0$. Write $\mathcal{H}=(\mathcal{F}, \mathcal{G})$ and denote by $\left(F_{1}, F_{2}, \ldots, F_{n-d}, G_{1}, \ldots, G_{d}\right)=(F, G)$ its associated formal holomorphic Taylor series at 0 . Let $h=\left(f_{1}, \ldots, f_{n-d}, g_{1}, \ldots, g_{d}\right)=(f, g)$ be the restriction of $\mathcal{H}$ to $M$ via the parametrisation of $M$. Note that $h$ is a smooth CR mapping from $M$ to $M^{\prime}$.

Recall that $\mathcal{H}$ is not totally degenerate at 0 if

$$
\operatorname{det}\left(\frac{\partial F_{j}}{\partial z_{k}}(z, 0)\right)_{1 \leq j, k \leq n-d} \not \equiv 0 .
$$

Also $\mathcal{H}$ is of finite multiplicity at 0 if

$$
\text { mult. } \mathcal{H}=\operatorname{dim}_{\mathbb{C}} \frac{\mathbb{C}[[z]]}{(F(z, 0))}<\infty .
$$

We shall see that the definitions above are independent of the choice of normal coordinates. We introduce the following definition.

Definition 1.4. Let $h$ be a smooth CR mapping from $M$ to $M^{\prime}$. We say that $h$ (or $\mathcal{H}$, if $h=\mathcal{H}_{\mid M}$ ) is transversally submersive if rank $g^{\prime}(0)=d$.

We shall see that $h$ is transversally submersive if and only if

$$
\operatorname{det}\left(\frac{\partial g_{j}}{\partial s_{k}}(0)\right)_{1 \leq j, k \leq d} \neq 0
$$

and that Definition 1.4 is independent of the choice of normal coordinates. For germs of real analytic generic submanifolds of codimension $d \geq 1$, we have the following theorems which extend the results obtained in [2], [3] and [4]. 
Theorem 1. Let $M$ and $M^{\prime}$ be two germs of real analytic generic submanifolds at 0 , and $\mathcal{H}$ (a germ at 0 of) a holomorphic map defined in a (germ of) wedge $\mathcal{W}$ with edge $M$, smooth in (the germ of) $\mathcal{W} \cup M$, and mapping $M$ into $M^{\prime}$, with $\mathcal{H}(0)=0$. Then $\mathcal{H}$ is the restriction of a holomorphic map in a neighborhood of 0 from $\mathbb{C}^{n}$ into $\mathbb{C}^{n}$ if one of the following conditions holds :

(1) $M$ is essentially finite, and $\mathcal{H}$ is transversally submersive.

(2) $M^{\prime}$ is essentially finite, and $\mathcal{H}$ is of finite multiplicity at 0.

(3) $M^{\prime}$ is essentially finite, and $\mathcal{H}$ is not totally degenerate at 0.

Moreover, (1) implies (2) implies (3).

Note that unlike the hypersurface case (cf. [4]) the conditions $M$ essentially finite and $\operatorname{det}\left(\partial G_{j} / \partial w_{k}\right)_{1 \leq j, k \leq d} \neq 0$, are not enough to guarantee $\mathcal{H}$ of finite multiplicity, as it is shown in the following example.

Example 1. In $\mathbb{C}^{3}$, consider $M$, of codimension 2, given by

$$
\begin{aligned}
& \operatorname{Im} w_{1}=\psi(z, \bar{z})-\operatorname{Re} w_{1} \psi(z, \bar{z}), \\
& \operatorname{Im} w_{2}=\operatorname{Re} w_{2} \psi(z, \bar{z}),
\end{aligned}
$$

$M^{\prime}$ given by

$$
\begin{aligned}
& \operatorname{Im} w_{1}^{\prime}=0, \\
& \operatorname{Im} w_{2}^{\prime}=\frac{1}{2 i}\left(z^{\prime} \bar{z}^{\prime 2}-\bar{z}^{\prime} z^{2}\right),
\end{aligned}
$$

$\mathcal{H}$ given by

$$
\mathcal{H}\left(z, w_{1}, w_{2}\right)=\left((1+z) w_{2}, w_{1} w_{2}-w_{2},-z(1+z) w_{2}^{3}\right),
$$

where $\psi$ is chosen so that $\mathcal{H}$ maps $M$ into $M^{\prime}$. By the implicit function theorem, it is possible to find such a $\psi$ with $\psi \not \equiv 0, \psi(z, 0) \equiv \psi(0, \bar{z}) \equiv 0$. In this example, $M$ is essentially finite as $\psi \neq \equiv 0, \operatorname{det}\left(\partial G_{j} / \partial w_{k}\right)_{1 \leq j, k \leq d} \neq 0$, but $\mathcal{H}$ is not of finite multiplicity.

Theorem 2. Let $h: M \longrightarrow M^{\prime}$ be a smooth CR mapping. Assume that $M$ is of finite type and essentially finite, and $h$ is transversally submersive. Then the map $h$ is real analytic.

Theorem 3. Let $M, M^{\prime}$ and $\mathcal{H}$ satisfy the hypothesis of Theorem 1. If either one of the following conditions holds:

(1) $M$ is essentially finite at 0 , and $\mathcal{H}$ is transversally submersive.

(2) $M^{\prime}$ is essentially finite at $0, \mathcal{H}$ is transversally submersive, and of finite multiplicity,

then ess. type $M=($ mult. $\mathcal{H})\left(\right.$ ess. type $\left.M^{\prime}\right)$,

with all three integers finite. 
Note that unlike the hypersurface case (cf. [4]) $M^{\prime}$ essentially finite at 0 and $\mathcal{H}$ of finite multiplicity do not imply $\mathcal{H}$ transversally submersive, as shown in the following example.

Example 2. Consider in $\mathbb{C}^{3} M$, of codimension 2, given by

$$
\operatorname{Im} w_{1}=z \bar{z}, \quad \operatorname{Im} w_{2}=z \bar{z},
$$

$M^{\prime}$ given by

$$
\operatorname{Im} w_{1}^{\prime}=\operatorname{Re} w_{2}^{\prime} z^{\prime} \bar{z}^{\prime}, \quad \operatorname{Im} w_{2}^{\prime}=z^{\prime} \bar{z}^{\prime},
$$

$\mathcal{H}$ given by

$$
\mathcal{H}\left(z, w_{1}, w_{2}\right)=\left(z, w_{1} w_{2}-\frac{1}{2} w_{1}^{2}, w_{2}\right)
$$

Here $M^{\prime}$ is essentially finite, $\mathcal{H}$ is of finite multiplicity, $\operatorname{det}\left(\partial G_{j} / \partial w_{k}\right)_{1 \leq j, k \leq d}$ $\neq 0$, but $\mathcal{H}$ is not transversally submersive.

2. Proof of Part 3 of Theorem 1. Write $w=s+i t$, and denote by $L_{j}, 1 \leq j \leq n-d$, the antiholomorphic vector fields tangent to $M$ (via the parametrisation) given by

$$
L_{j}=\frac{\partial}{\partial \bar{z}_{j}}-i \sum_{k=1}^{d}\left(\frac{\partial \varphi_{k}}{\partial \bar{z}_{j}}\right) M_{k},
$$

with

$$
M_{k}=\sum_{k^{\prime}=1}^{d} \mu_{k^{\prime}} \frac{\partial}{\partial s_{k^{\prime}}},
$$

where $\left(\mu_{k^{\prime} k}\right)_{k, k^{\prime}=1, \ldots, d}$ is the inverse of the Jacobian matrix of $s+i \varphi(z, \bar{z}, s)$ with respect to $s$. Let $\alpha=\left(\alpha_{1}, \ldots, \alpha_{n-d}\right) \in \mathbb{Z}^{n-d}$ and put $L^{\alpha}=L_{1}^{\alpha_{1}} \ldots L_{n-d}^{\alpha_{n-d}}$. We have the following lemmas.

Lemma 2.2. Let $M, M^{\prime}$ and $\mathcal{H}$ satisfy the hypothesis of Theorem 1. Then

$$
L^{\alpha} \bar{f}(0)=\left(\frac{\partial}{\partial \bar{z}}\right)^{\alpha} \bar{F}(0), \quad L^{\alpha} \bar{G}(0)=\left(\frac{\partial}{\partial \bar{z}}\right)^{\alpha} \bar{g}(0)=0 .
$$

This is easily shown by using the fact that $\varphi(0, \zeta, 0) \equiv 0$ in normal coordinates. 
Lemma 2.4. Let $M, M^{\prime}$ and $\mathcal{H}$ satisfy the hypothesis of Theorem 1. If $\mathcal{H}$ is not totally degenerate, then there exists a multi-index $\alpha$ such that

$$
L^{\alpha}\left(\operatorname{det}\left(L_{j} \bar{f}_{k}\right)_{1 \leq j, k \leq n-d}\right)(0) \neq 0 .
$$

This is immediate from the definition and Lemma 2.2.

Let $M^{\prime}$ given in normal coordinates by $\operatorname{Im} w^{\prime}=\psi\left(z^{\prime}, \overline{z^{\prime}}\right.$, Re $\left.w^{\prime}\right)$, with $z^{\prime} \in$ $\mathbb{C}^{n-d}, w^{\prime} \in \mathbb{C}^{d}, \psi=\left(\psi_{1}, \ldots, \psi_{d}\right)$, where $\psi_{j}, 1 \leq j \leq d$, are real-valued convergent power series and $\psi\left(z^{\prime}, 0, w^{\prime}\right) \equiv 0$. Solving for $\bar{w}^{\prime}$, it is easily shown that $M^{\prime}$ is also given by

$$
\bar{w}^{\prime}=Q\left(z^{\prime}, \bar{z}^{\prime}, w^{\prime}\right)=w^{\prime}+R\left(z^{\prime}, \bar{z}^{\prime}\right)+S\left(z^{\prime}, \bar{z}^{\prime}, w^{\prime}\right),
$$

with $Q=\left(Q_{j}\right)_{1 \leq j \leq d}, R=\left(R_{j}\right)_{1 \leq j \leq d}, S=\left(S_{j}\right)_{1 \leq j \leq d}$, where $Q_{j}, R_{j}, S_{j}$ are convergent power series and $Q\left(z^{\prime}, 0, w^{\prime}\right) \equiv Q\left(0, \bar{z}^{\prime}, w^{\prime}\right) \equiv w^{\prime}, S\left(z^{\prime}, \bar{z}^{\prime}, 0\right) \equiv 0$.

Let $U$ be an open neighborhood of 0 in $\mathbb{R}^{2 n}$, and let

$$
\begin{gathered}
\Psi: U \subset \mathbb{R}^{2 n} \longrightarrow \mathbb{C}^{n} \\
(z, s, t) \longrightarrow(z, s+i t+i \varphi(z, \bar{z}, s+i t)) .
\end{gathered}
$$

Define the following wedges:

$$
\begin{gathered}
W_{\Gamma}=\left\{(z, s, t) \in \mathbb{R}^{2 n} \mid t \in \Gamma\right\}, \\
\mathcal{W}_{\Gamma}=\left\{(z, s+i t+i \varphi(z, \bar{z}, s)) \in \mathbb{C}^{n} \mid t \in \Gamma\right\} .
\end{gathered}
$$

Let $\Gamma^{\prime}$ be an open convex cone with vertex at the origin whose closure is contained in $\Gamma \cup\{0\}$. We have (in the sense of germs at 0 )

$$
\mathcal{W}_{\Gamma^{\prime}} \subset \Psi\left(W_{\Gamma}\right), \Psi\left(W_{\Gamma^{\prime}}\right) \subset \mathcal{W}_{\Gamma} .
$$

It is then clear that since $\mathcal{H}$ is (a germ at 0 of) a holomorphic map in (the germ of) $\mathcal{W}_{\Gamma}, f_{1}, \ldots, f_{n-d}$ and $g_{1}, \ldots, g_{d}$ extend continuously with respect to all its variables in the wedge $W_{\Gamma^{\prime}}$ (in the sense of germs at 0 ) in such a way that this extension is holomorphic with respect to $s+i$. It follows that $\bar{f}_{1}, \ldots, \bar{f}_{n-d}, \bar{g}_{1}, \ldots$, $\bar{g}_{d}$ extend continuously with respect to all its variables in the wedge $W_{-\Gamma^{\prime}}$ (in the sense of germs at 0) in such a way that these extensions are holomorphic with respect to $s+i t$. We have the following propositions.

Proposition 2.7. Let $M, M^{\prime}$ and $\mathcal{H}$ satisfy the hypothesis of Theorem 1. If $\mathcal{H}$ is not totally degenerate then $R(f, \bar{f})$ satisfies the following equation

$$
R_{\zeta^{\alpha}}(f, \bar{f})+K_{\alpha}\left(f, u_{\alpha}\right)=0,
$$

where $\alpha$ is any multi-index, with $|\alpha| \geq 1, K_{\alpha}$ is a d-tuple of holomorphic functions in a neighborhood of $\left(0, u_{\alpha}(0)\right), u_{\alpha}$ is a finite set of functions which extend continuously with respect to all its variables in an open set $O=W_{-\Gamma^{\prime}}$ (in the sense of germs at 0$), O$ independent of the multi-index $\alpha$, in such a way that these extensions are holomorphic with respect to $s+i t$ in $O$, and $K_{\alpha}\left(z, u_{\alpha}(0)\right) \equiv 0$, $z \in \mathbb{C}^{n-d}$. 
Proof. As $\mathcal{H}(M) \subset M^{\prime}$, we have

$$
\bar{g}_{1}=g_{1}+R_{1}(f, \bar{f})+S_{1}(f, \bar{f}, g)
$$

$$
\bar{g}_{d}=g_{d}+R_{d}(f, \bar{f})+S_{d}(f, \bar{f}, g)
$$

We will prove Proposition 2.7 for the case $|\alpha|=1$. Applying $L_{j}$ defined by (2.1) to (2.8), and using the fact that

$$
L_{j} g=L_{j} f=0,
$$

we obtain

$$
L_{j} \bar{g}_{1}=\sum_{l=1}^{n-d}\left(R_{1 \zeta_{\ell}}(f, \bar{f})+S_{1 \zeta_{\ell}}(f, \bar{f}, g)\right) L_{j} \bar{f}_{l}
$$

$$
L_{j} \bar{g}_{d}=\sum_{l=1}^{n-d}\left(R_{d \zeta_{\ell}}(f, \bar{f})+S_{d \zeta_{\ell}}(f, \bar{f}, g)\right) L_{j} \bar{f}_{l} .
$$

Considering the $(n-d) \times d$ equations given by $(2.9)$ with unknown $R_{\zeta_{k}}(f, \bar{f})+$ $S_{\zeta_{k}}(f, \bar{f}, g)$ and using Cramer's rule, we obtain

$$
\operatorname{det}\left(L_{j} \bar{f}_{l}\right)\left(R_{\zeta_{k}}(f, \bar{f})+S_{\zeta_{k}}(f, \bar{f}, g)\right)=h_{k}
$$

where $h_{k}$ is a d-tuple of functions which extend continuously with respect to all its variables in the wedge $W_{-\Gamma^{\prime}}$ (in the sense of germs at 0 ), in such a way that these extensions are holomorphic with respect to $s+i t$.

Choose $\beta_{0}$ to be a multi-index of minimal length satisfying (2.5). Applying $L^{\beta_{0}}$ to both sides of (2.10), we obtain

$$
\begin{aligned}
& L^{\beta_{0}}\left(\operatorname{det} L_{j} \bar{f}_{l}\right)\left(R_{\zeta_{k}}(f, \bar{f})+S_{\zeta_{k}}(f, \bar{f}, g)\right) \\
& \quad \sum_{\substack{\gamma_{1}+\gamma_{2}=\beta_{0} \\
\gamma_{2} \neq 0}} c L^{\gamma_{1}}\left(\operatorname{det} L_{j} \bar{f}_{l}\right)\left(L^{\gamma_{2}}\left(R_{\zeta_{k}}(f, \bar{f})+S_{\zeta_{k}}(f, \bar{f}, g)\right)\right)=L^{\beta_{0}} h_{k} .
\end{aligned}
$$

Dividing (2.11) by $L^{\beta_{0}}\left(\operatorname{det} L_{j} \bar{f}_{l}\right)$, we obtain

$$
\begin{array}{r}
R_{\zeta_{k}}(f, \bar{f})+S_{\zeta_{k}}(f, \bar{f}, g)=-\sum_{\substack{\gamma_{1}+\gamma_{2}=\beta_{0} \\
\gamma_{2} \neq 0}} c \frac{L^{\gamma_{1}}\left(\operatorname{det} L_{j} \bar{f}_{l}\right)}{L^{\beta_{0}}\left(\operatorname{det} L_{j} \bar{f}_{l}\right)} L^{\gamma_{2}}\left(R_{\zeta_{k}}(f, \bar{f})\right. \\
\left.+S_{\zeta_{k}}(f, \bar{f}, g)\right)+\frac{L^{\beta_{0}} h_{k}}{L^{\beta_{0}}\left(\operatorname{det} L_{j} \bar{f}_{l}\right)} .
\end{array}
$$


Taking the complex conjugate of (2.8), we obtain an expression for $g$ which depends of $f, \bar{f}$ and $\bar{g}$. Making use of that, we can rewrite (2.12) as

$$
R_{\zeta_{k}}(f, \bar{f})+K_{k}(f, u)=0,
$$

where

$$
u=\left\{\frac{L^{\beta_{0}} h_{k}}{L^{\beta_{0}}\left(\operatorname{det} L_{j} \bar{f}_{l}\right)}, \bar{f}, \bar{g}, \frac{L^{\gamma_{1}}\left(\operatorname{det} L_{j} \bar{f}_{\ell}\right)}{L^{\beta_{0}}\left(\operatorname{det} L_{j} \bar{f}_{l}\right)}, L^{\alpha} \bar{f}, L^{\beta} \bar{g},|\alpha|,|\beta| \leq\left|\beta_{0}\right|\right\},
$$

where $K_{\alpha}$ is a d-tuple of holomorphic functions in a neighborhood of $(0, u(0))$. All the functions in the set $u$ extend continuously with respect to all its variables in the wedge $W_{-\Gamma^{\prime}}$ (in the sense of germs at 0 ), in such a way that these extensions are holomorphic with respect to $s+i t$. By minimality of $\beta_{0}, K_{\alpha}(z, u(0)) \equiv 0$, $z \in \mathbb{C}^{n-d}$. This achieves the proof of Proposition 2.7 for the case $|\alpha|=1$.

For the general case, i.e., for a multi-index $\alpha$ of any length, we observe that

$$
R_{\zeta^{\alpha}}(f, \bar{f})+S_{\zeta^{\alpha}}(f, \bar{f}, g)=\frac{d_{\alpha}}{\left[\operatorname{det}\left(L_{j} \bar{f}_{l}\right)\right]^{2|\alpha|-1}}
$$

where $d_{\alpha}$ is a d-tuple of functions which extend continuously with respect to all its variables in the wedge $W_{-\Gamma^{\prime}}$ (in the sense of germs at 0 ), in such a way that these extensions are holomorphic with respect to $s+i t$. The details of the proof are similar to the ones of the proof of Proposition 3.15 in [16].

Proposition 2.13. Let $M, M^{\prime}$ and $\mathcal{H}$ satisfy the hypothesis of Theorem 1 , with $M^{\prime}$ essentially finite at 0 and $\mathcal{H}$ not totally degenerate. Then each $f_{j}$ satisfies a polynomial equation with analytic coefficients depending on functions which extend continuously with respect to all its variables in the wedge $W_{-\Gamma^{\prime}}$ (in the sense of germs at 0 ), in such a way that these extensions are holomorphic with respect to $s+i t$.

Proof. Let $M^{\prime}$ given by (2.6). Write $R_{k}(z, \bar{z})=\sum_{\alpha} b_{\alpha}^{k}(z) \bar{z}^{\alpha}$. Using the fact that $M^{\prime}$ is essentially finite and the Nullstellensatz, we can find $N$ and $r$ such that

$$
z_{j}^{N}=\sum_{|\alpha|, k=1}^{r} c_{j \alpha}^{k}(z) b_{\alpha}^{k}(z), \quad 1 \leq j \leq n-d,
$$

with $c_{j \alpha}^{k}(z)$ convergent power series. We also have

$$
R_{k \zeta^{\alpha}}(z, \bar{z})=\alpha ! b_{\alpha}^{k}(z)+\sum_{|h| \geq 1} d_{\alpha h}^{k}(z) \bar{z}^{h}, \quad 1 \leq k \leq d
$$


with $d_{\alpha h}^{k}(z)$ convergent power series. As $f(0)=0$, we may substitute $f_{j}$ in $(2.14)$ and then get

$$
f_{j}^{N}=\sum_{|\alpha|, k=1}^{r} c_{j \alpha}^{k}(f) b_{\alpha}^{k}(f) .
$$

Using Proposition $2.7,(2.15)$ and $(2.16)$, we obtain

$$
f_{j}^{N}+H_{j}(f, u)=0
$$

where $H_{j}$ is a holomorphic function in a neighborhood of $(0, u(0))$, with $u$ a set of functions which extend continuously with respect to all its variables in the wedge $W_{-\Gamma^{\prime}}$ (in the sense of germs at 0 ), in such a way that these extensions are holomorphic with respect to $s+i t$, and $H_{j}(z, u(0)) \equiv 0$. Using $(2.17)$, the Weierstrass Preparation Theorem and the classical Newton Theorem for symmetric functions, we claim that each $f_{j}$ satisfies a polynomial equation with holomorphic coefficients depending on the set of functions $u$. The proof of the claim follows by inspecting the very end of Lemma 6.1 in [3]. Hence we get the desired conclusion. holds:

Proposition 2.18. There exists an open set $O \subset \mathbb{C}^{n}$ such that the following

(i) $O=W_{-\Gamma^{\prime}}$ in the sense of germs at 0 .

(ii) For every multi-index $\alpha$, there exist functions $u_{\alpha}^{k}(z, \bar{z}, s, t), v_{\alpha}^{k}(z, \bar{z}, s, t)$ defined in $\bar{O}$, smooth with respect to $(s, t)$, holomorphic with respect to $s+i t$ in $O$, such that

$$
Q_{k \zeta^{\alpha}}(f, \bar{f}, g)(z, \bar{z}, s)=\frac{u_{\alpha}^{k}(z, \bar{z}, s, 0)}{v_{\alpha}^{k}(z, \bar{z}, s, 0)},
$$

with $v_{\alpha}^{k}(z, \bar{z}, s, 0) \not \equiv 0$ as a function of $s$.

The proof is similar to that of Lemma (5.3) in [3] and is left to the reader.

We consider $B(0, R) \subset \mathbb{R}_{(s, t)}^{2 d}$ the open ball centered at 0 with radius $R$, and $V_{\Gamma} \subset \mathbb{R}_{(s, t)}^{2 d}$ the wedge with edge $t=0$ in the direction $\Gamma$. Put $S=V_{\Gamma} \cap B(0, R)$. We need the following lemmas.

Lemma 2.19. Let $b_{1}, \ldots, b_{p}$ be smooth functions in $\bar{S}$ and let $h$ be a continuous function defined in $S$ which satisfies the following polynomial equation:

$$
h^{p}+\sum_{i=1}^{p} b_{i} h^{p-i}=0 .
$$

Then we have the following:

(i) $\left|h(s, t)-h\left(s^{\prime}, t^{\prime}\right)\right| \leq C\left|(s, t)-\left(s^{\prime}, t^{\prime}\right)\right|^{1 / p}$, with $C>0$, for $(s, t),\left(s^{\prime}, t^{\prime}\right) \in S$,

(ii) $h$ extends continuously to $\bar{S}$. 
Proof. Since $b_{j}$ are smooth in $\bar{S}$, we can find $K>0$ such that

$$
\begin{gathered}
\left|b_{j}(s, t)\right| \leq K^{j} \\
\left|b_{j}(s, t)-b_{j}\left(s^{\prime}, t^{\prime}\right)\right| \leq K^{j}\left|(s, t)-\left(s^{\prime}, t^{\prime}\right)\right| .
\end{gathered}
$$

Since we work in $\bar{S}$, with $\Gamma$ an open convex cone, we can apply the same proof as the one of Lemma 2.5 of Chapter 4 in [15] to conclude Part (i).

To prove part (ii), we take a sequence $\left(s_{n}, t_{n}\right)$ such that $\left(s_{n}, t_{n}\right) \longrightarrow(s, 0)$. We have to show that $\lim _{\left(s_{n}, t_{n}\right) \longrightarrow(s, 0)} h\left(s_{n}, t_{n}\right)$ exists. By Part (i), we have

$$
\left|h\left(s_{n}, t_{n}\right)-h\left(s_{m}, t_{m}\right)\right| \leq C\left|\left(s_{n}, t_{n}\right)-\left(s_{m}, t_{m}\right)\right| .
$$

We conclude that $h\left(s_{n}, t_{n}\right)$ is a Cauchy sequence. Hence $\lim _{\left(s_{n}, t_{n}\right) \rightarrow(s, 0)} h\left(s_{n}, t_{n}\right)$ exists. Using again Part (i), we obtain that this limit is independent of the choice of the sequence $\left(s_{n}, t_{n}\right)$. This achieves the proof of Lemma 2.19.

Lemma 2.20. Let $u$, $v$, with $v \not \equiv 0$, be two functions defined in $\bar{S}$, and satisfying

(i) $u, v$ are smooth in $\bar{S}$, holomorphic in $S$.

(ii) $h(s)=u(s, 0) / v(s, 0)$ is smooth for $(s, 0) \in \bar{S}$.

(iii) $h(s)$ satisfies a polynomial equation of the form

$$
h(s)^{p}+\sum_{j=1}^{p} a_{j}(s) h(s)^{p-j}=0,
$$

where $a_{j}$ are smooth functions in $\bar{S}$, holomorphic in $S$.

Then $h$ extends holomorphically to $S$ as $\frac{u}{v}$ and $\frac{u}{v}$ is smooth in $\bar{S}$.

Proof. Let $\left(s_{0}, 0\right) \in \bar{S}$ such that $v\left(s_{0}, 0\right) \neq 0$ and let $\widetilde{h}(w)$ be the meromorphic function in $S$ defined by $\frac{u(w)}{v(w)}$. Using (2.21), we conclude that

$$
\widetilde{h}(w)^{p}+\sum_{j=1}^{p} a_{j}(w) \widetilde{h}(w)^{p-j}=0
$$

by analytic continuation, for $w$ in $S$, except for the set $\{w \in S \mid v(w)=0\}$. Since the coefficients $a_{j}$ are bounded in $S,(2.22)$ implies that $\widetilde{h}(w)$ is bounded in $S$. Therefore, using the extended Riemann removable singularities theorem, we can conclude that $\widetilde{h}(w)$ is extendable to a holomorphic function is $S$. From Lemma 2.19 , we get that $\widetilde{h}(w)$ has a boundary value which is a continuous function. Let $(s, 0) \in \bar{S}$ such that $v(s, 0)=0$. As $v \not \equiv 0$, we can find a sequence $\left(s_{n}, 0\right) \in \bar{S}$ with $v\left(s_{n}, 0\right) \neq 0$ and $s_{n} \rightarrow s$. Therefore, as $\widetilde{h}\left(s_{n}\right)=h\left(s_{n}\right), \widetilde{h}(s)=h(s)$. Therefore, since the boundary value $h(s)$ of $\widetilde{h}(w)$ is $C^{\infty}$, we reach the conclusion, using Lemma 2.4 in [2] . This achieves the proof of Lemma 2.20. 
Proof of Part (3) of Theorem 1. Using Proposition 2.13 and Lemma 8.11 in [1], we obtain that each $Q_{k \zeta^{\alpha}}(f, \bar{f}, g)$, satisfies a polynomial relation with coefficients which are analytic functions depending on functions which extend continuously with respect to all its variables in an open set $O$ equivalent to the wedge $W_{-\Gamma^{\prime}}$ in the sense of germs at $0, O$ independent of the multi-index $\alpha$, in such a way that these extensions are holomorphic with respect to $s+i t$ in $O$. Hence, using Proposition 2.18, Lemma 2.20 and Lemma 8.11 in [1], we conclude that each $Q_{k \zeta^{\alpha}}(f, \bar{f}, g)$ extends in $\bar{O}$, smoothly with respect to $(s, t)$, in such a way that the extension is holomorphic with respect to $s+i t$ in $O$, with

$$
\left|Q_{k \zeta^{\alpha}}(f, \bar{f}, g)(z, \bar{z}, s+i t)\right| \leq C^{\alpha} \alpha !
$$

Therefore, following the proof of Theorem 6.1 in [2], we conclude that

$$
Q(f, \lambda, g)(z, \bar{z}, s)=\sum_{\alpha=0}^{\infty} \frac{(\lambda-\bar{f})^{\alpha}}{\alpha !} Q_{\zeta^{\alpha}}(f, \bar{f}, g)(z, \bar{z}, s)
$$

extends in $W_{\Gamma^{\prime}}$ and $W_{-\Gamma^{\prime}}$ in the sense of germs at 0 , smoothly with respect to $(s, t)$, in such a way that the extension is holomorphic with respect to $s+i t$ for $\lambda$ small enough. Using the Edge of the Wedge Theorem, we conclude that $Q(f, \lambda, g)$ extends in a neighborhood of 0 in $\mathbb{C}^{n}$, holomorphically with respect to $s+i t$, in such a way that its extension is a bounded and measurable function of all its variables. Putting $\lambda=0$ in (2.23), and using (2.6), the same will be true for $g$. As $g$ is a CR mapping, we claim this implies that $\mathcal{G}$ is the restriction of a holomorphic map in the neighborhood of 0 from $\mathbb{C}^{n}$ to $\mathbb{C}^{d}$. This is shown by using a slight modification of Corollary 2.1 and Lemma 2.2 in [2]. Put $Q(f, \lambda, g)(x, y, s)=F(\lambda, x, y, s)$. As $Q(f, \lambda, g)$ is a CR mapping, $F(\lambda, x, y, s)$ is real analytic of $x, y, s$, for the same reasons as below. Using (2.6), we get

$$
g(x, y, s)+R(f, \lambda)(x, y, s)+S(f, \lambda, g)(x, y, s)-F(\lambda, x, y, s)=0 .
$$

Differentiating with respect to $\lambda$ enough times, we get

$$
\alpha ! b_{\alpha}(f)+\sum_{|h| \geq 1} d_{\alpha h}(f) \bar{f}^{h}+S_{\lambda^{\alpha}}(f, \lambda, g)-F_{\lambda^{\alpha}}=0 .
$$

Using the same arguments as in the proof of Proposition 2.13, and using the fact that $F(\lambda, 0,0,0) \equiv 0$, we obtain equations of the form

$$
f_{j}^{N}+H_{\alpha}\left(f, \lambda, g, F_{\lambda^{\alpha}}\right) \equiv 0
$$

with $H_{\alpha}(z, 0,0,0) \equiv 0, z \in \mathbb{C}^{n-d}$, and hence equations of the form

$$
f_{j}^{N}+\sum_{k=1}^{N} b_{j}\left(\lambda, g, F_{\lambda^{\alpha}}\right) f_{j}^{N-k} \equiv 0,
$$

with $b_{j}$ analytic in a neighborhood of 0 . Putting $\lambda=0$ and using Lemma (6.1) in [2], we obtain that $\mathcal{F}$ is the restriction of a holomorphic map from a neighborhood of 0 in $\mathbb{C}^{n}$ to $\mathbb{C}^{n-d}$. This achieves the proof of part (3) of Theorem 1. 
3. Generic submanifolds of essential type and mappings of finite multiplicity. In this section, the theorems and propositions we state have been proved for the hypersurface case (cf. [3]). Their proofs are very similar, so we will sketch them and refer very often the reader to [3].

Let $M$ be a germ of a real analytic generic submanifold given in normal coordinates by (1.1). Similarily we assume that $M^{\prime}$ is given in normal coordinates by

$$
\operatorname{Im} w^{\prime}=\psi\left(z^{\prime}, \overline{z^{\prime}}, \operatorname{Re} w^{\prime}\right),
$$

with $z^{\prime} \in \mathbb{C}^{n-d}, w^{\prime} \in \mathbb{C}^{d}, \psi=\left(\psi_{1}, \ldots, \psi_{d}\right)$, where $\psi_{j}, 1 \leq j \leq d$, are real valued convergent power series and $\psi\left(z^{\prime}, 0, w^{\prime}\right) \equiv 0$.

Proposition 3.2. Let $M, M^{\prime}$ and $\mathcal{H}$ satisfy the hypothesis of Theorem 1. Then the following is true:

(i) $\operatorname{det}\left(\left(\partial G_{j} / \partial w_{k}\right)(0)\right)_{1 \leq j, k \leq d}=\operatorname{det}\left(\left(\partial g_{j} / \partial s_{k}\right)(0)\right)_{1 \leq j, k \leq d} \in \mathbb{R}$.

(ii) $G(z, 0) \equiv 0$.

(iii) There exist formal power series $\beta_{j k}(z, \zeta), 1 \leq j, k \leq d$, such that

$$
\sum_{k=1}^{d}, \beta_{j k}(z, \zeta) \varphi_{k}(z, \zeta, 0)=\psi_{j}(F(z, 0), \bar{F}(\zeta, 0), 0), \quad 1 \leq j \leq d .
$$

Furthermore, if $\operatorname{det}\left(\left(\partial G_{j} / \partial w_{k}\right)(0)\right)_{1 \leq j, k \leq d} \neq 0$, then $\operatorname{det}\left(\beta_{j k}(0)\right)_{1 \leq j, k \leq d} \neq 0$.

Proof. We have $\left.g_{j}(z, \bar{z}, s) \sim G_{j}(z, \bar{z}, w)\right|_{w=s+i \varphi(z, \bar{z}, s)}$. Hence, as we work in normal coordinates,

$$
\frac{\partial g_{j}}{\partial s_{k}}(0)=\frac{\partial G_{j}}{\partial w_{k}}(0), \quad 1 \leq j, k \leq d
$$

As $\mathcal{H}(M) \subset M^{\prime}$,

$$
\frac{g_{j}-\bar{g}_{j}}{2 i}=\psi_{j}\left(f, \bar{f}, \frac{g+\bar{g}}{2}\right), \quad 1 \leq j \leq d,
$$

and hence, as we work in normal coordinates, this implies $\left(\partial / \partial s_{k}\right)\left(\operatorname{Im} g_{j}\right)(0)=0$. This achieves the proof of (i). Part (ii) is an immediate consequence of Lemma 2.2. Using the hypothesis (i) and (ii), we get

$$
\begin{aligned}
& \sum_{k=1}^{d} c_{j k}(z, \zeta) \varphi_{k}(z, \zeta, 0) \\
& \quad=\left.\psi_{j}\left(F, \bar{F}, \frac{G+\bar{G}}{2}\right)\right|_{(z, i \varphi(z, \zeta, 0))} \\
& \quad=\psi_{j}(F(z, 0), \bar{F}(\zeta, 0), 0)+\sum_{\ell=1}^{d} d_{j \ell}(z, \zeta) \varphi_{\ell}(z, \zeta, 0), \quad 1 \leq j \leq d,
\end{aligned}
$$


where $c_{j k}(z, \zeta), 1 \leq j, k \leq d$ and $d_{j \ell}(z, \zeta), 1 \leq j, \ell \leq d$ are formal power series with

$$
\begin{aligned}
& \operatorname{det}\left(c_{j k}(0)\right)_{1 \leq j, k \leq d}=2^{d} \operatorname{det}\left(\frac{\partial G_{j}}{\partial w_{k}}(0)\right)_{1 \leq j, k \leq d} \\
& \operatorname{det}\left(d_{j \ell}(0)\right)_{1 \leq j, \ell \leq d}=0 .
\end{aligned}
$$

By combining the term in the left hand side with the second term on the right hand side, we obtain (3.3). This achieves the proof of Proposition 3.2.

The following corollaries are an immediate consequence of Part (ii) of Proposition 3.2 .

Corollary 3.4. $h$ is transversally submersive if and only if

$$
\operatorname{det}\left(\frac{\partial g_{j}}{\partial s_{k}}(0)\right)_{1 \leq j, k \leq d} \neq 0
$$

Corollary 3.5. Definition 1.4 is independent of the choice of normal coordinates. The same is true for $\mathcal{H}$ not totally degenerate and $\mathcal{H}$ of finite multiplicity.

Theorem 3.6. Let $M, M^{\prime}$ and $\mathcal{H}$ satisfy the hypothesis of Theorem 1. Then the following is true:

(i) If $\mathcal{H}$ is of finite multiplicity and $M^{\prime}$ is essentially finite, then $M$ is essentially finite.

(ii) If $M$ is essentially finite and $\mathcal{H}$ is transversally submersive, then $\mathcal{H}$ is of finite multiplicity and $M^{\prime}$ is essentially finite.

Remark 3.7. For the proof of Theorem 3.6, we shall use the fact that a finite holomorphic mapping is open and vanishes only at zero in a neighborhood of zero.(See [12].)

Proof of Part (i) of Theorem 3.6. Using Lemma 3.19 in [3] and Part (3) of Theorem 1, we conclude that $F$ is a $(n-d)$-tuple of convergent power series. Assume by contradiction that there exists $z_{0} \neq 0$ such that

$$
\varphi_{k}\left(z_{0}, \zeta, 0\right)=0, \quad \forall \zeta \in \mathbb{C}^{n-d}, 1 \leq k \leq d .
$$

Using (3.3) and Remark 3.6, we may conclude that there exists $z_{0}^{\prime} \neq 0$ such that

$$
\psi_{j}\left(z_{0}^{\prime}, \zeta^{\prime}, 0\right)=0, \quad \forall \zeta^{\prime} \in \mathbb{C}^{n-d}, 1 \leq j \leq d,
$$

contradicting the essential finitness of $M^{\prime}$. This completes the proof of (i). 
Proof of Part (ii) of Theorem 3.6. Using Part (iii) of Proposition 3.2, and the normal coordinates, we conclude that

$$
\left(a_{\alpha}^{j}(z)\right) \subset(F(z, 0)), \quad 1 \leq j \leq d .
$$

This shows that $\mathcal{H}$ is of finite multiplicity. It remains to prove that $M^{\prime}$ is essentially finite. Let $F^{r}(z, 0)$ ( resp. $\left.\beta_{j k}^{r}(z, \zeta)\right)$ be the Taylor polynomials of degree $r$ of $F(z, 0)$ (resp. $\beta_{j k}(z, \zeta)$ ). Using Part (iii) of Proposition 3.2, there exists a d-tuple of analytic functions $\varphi^{(r)}(z, \zeta)$ such that

$$
\sum_{k=1}^{d} \beta_{j k}^{r}(z, \zeta) \varphi_{k}^{(r)}(z, \zeta)=\psi_{j}\left(F^{r}(z, 0), \overline{F^{r}}(\zeta, 0), 0\right), \quad 1 \leq j \leq d .
$$

Making use of the Noetherian Theorem, Lemma 4.3 in [3], and Remark 3.7, we may conclude that $M^{\prime}$ is essentially finite, by using the same arguments as in the proof of Theorem 3 in [3]. This achieves the proof of Theorem 3.6.

\section{Proof of Theorems 1, 2 and 3.}

Proof of Theorem 1. Using (ii) of Theorem 3.6, we get that (1) implies (2), which implies (3), using Lemma 3.19 in [3]. The proof of Theorem 1 is then complete.

Proof of Theorem 3. By (ii) of Theorem 3.6, (1) implies (2). Therefore, by Theorem $1, F$ is a finite holomorphic mapping. Hence, making use of part (iii) of Proposition 3.2, Lemma 4.7 in [3] as well as Remark 3.7, we obtain an equality in $\mathbb{C}[[z]]$ between the ideals $\left(\varphi_{k}(z, \zeta, 0) ;|\zeta|<\varepsilon, 1 \leq k \leq d\right)$, and the ideals $\left(\psi_{j}\left(z, \zeta^{\prime}, 0\right) ;\left|\zeta^{\prime}\right|<\varepsilon, 1 \leq j \leq d\right)$, provided $\varepsilon>0$ is small enough. Using Lemmas 4.7 and 4.5 in [3], this will show the equality (1.6). It remains to show that (2) implies (1.6). This is easily shown by using Part (i) of Theorem 3.6. This achieves the proof of Theorem 3.

The following corollary is an immediate consequence of (1.6).

Corollary 4.1. The number ess. type $M$ defined by (1.3) is independent of the choice of normal coordinates.

Proof of Theorem 2. As $M$ is of finite type, the theory of Tumanov [19] shows that $h$ extends smoothly to a holomorphic mapping $\mathcal{H}$ defined in a wedge $\mathcal{W}$ with edge $M$. Part (1) of Theorem 1 allows us to conclude that $h$ is real analytic. This achieves the proof of Theorem 2 . 


\section{REFERENCES}

[1] M. S. Baouendi, S. Bell, And L. P. Rothshild, Mappings of three-dimensional CR manifolds and their holomorphic extension, Duke Math. J. 56, 503-530.

[2] M. S. Baouendi, H. Jacobowitz, F. Treves, On the analyticity of $C R$ mappings, Ann. of Math. 122, 365-400.

[3] M. S. BAOUendi And L. P. RothsChild, Germs of CR maps between real analytic hypersurfaces, Invent. Math. 93, 481-500.

[4] M. S. BAOUendi And L. P. Rothschild, Geometric properties of smooth and holomorphic mappings between hypersurfaces in complex space, J. Diff. Geom. 31, 473-499.

[5] M. S. BaOUendi And L. P. Rothschild, A general reflection principle in $\mathbb{C}^{2}$, Journal of Functional Analysis 99, 409-442.

[6] S. BELL, Analytic hypoellipticity of the $\bar{\partial}$-Neumann problem and extendability of holomorphic mappings, Acta Math. 147, 109-116.

[7] J. D'ANGELO, The notion of formal essential finitness for smooth real hypersurfaces, Indiana J. Math. 36, 897-903.

[8] T. Bloom AND I. Graham, On type conditions for generic submanifolds of $\mathbb{C}^{n}$, Inventiones Math. 40, 217-243.

[9] K. Diederich And J. E. Fornaess, Proper holomorphic mappings between real analytic pseudoconvex domains in $\mathbb{C}^{n}$, Math. Ann. 282, 681-700.

[10] K. Diederich AND S. M. WeBSTER, A reflection principle for degenerate real hypersurfaces, Duke Math. J. 47, 835-843.

[11] D. Eisenbud AND H. Levine, An algebraic formula for the degree of a $C^{\infty}$ map germ, Annals of Math. 106, 19-44.

[12] P. GRIffiths AND J. HARRIS, Principles of Algebraic Geometry, Wiley-Interscience, New-York.

[13] J. J. KoHN, Boundary behavior of $\bar{\partial}$ on weakly pseudoconvex manifolds of dimension two, J. Differential Geom. 6, 523-542.

[14] H. LEWY, On the boundary behavior of holomorphic mappings, Acad. Naz. Lincei 35, $1-8$.

[15] B. Malgrange, Ideals of differentiable functions, Tata Institute of Fundamental Research, Bombay, Oxford University Press.

[16] F. MEYLAN, A reflection principle in complex space for a class of hypersurfaces and mappings, Pacific Journal of Mathematics (to appear).

[17] Extension of germs of smooth CR diffeomorphisms between non essentially finite hypersurfaces hypersurfaces in $\mathbb{C}^{3}$, C. R. Acad. Sci. Paris, 319 Serie I, 827-830.

[18] S. I. PINCHUK, On proper holomorphic mappings of strictly pseudoconvex domains, Siberian Math. J. 15, 909-917.

[19] A. E. Tumanov, Extending CR functions on manifolds of finite type to a wedge (Russian), Mat. Sbornik 136, 128-139; also Math. USSR Sb. 64 (1989), 129-140.

Section de Mathématiques

Université de Genève

2-4, rue du Lièvre

Case postale 240

CH-1211 Genève 24

Switzerland

Received: November 1st, 1994; revised: August 8th, 1995. 